\title{
Trade-offs in the visible spectrograph of the ELT instrument MOSAIC
}

\section{A. W. Janssen, E. Sokolova, J. Pragt, J. Kragt, Ramon Navarro, et al.}

A. W. Janssen, E. Sokolova, J. Pragt, J. Kragt, Ramon Navarro, Niels Tromp, E. Fitzsimons, M. Rodrigues, M. Larrieu, A. Kelz, T. Morris, P. Jagourel, F. Chemla, L. Kaper, G. Dalton, " Trade-offs in the visible spectrograph of the ELT instrument MOSAIC," Proc. SPIE 10702, Groundbased and Airborne Instrumentation for Astronomy VII, 107029F (10 July 2018); doi: 10.1117/12.2313302

SPIE Event: SPIE Astronomical Telescopes + Instrumentation, 2018, Austin, Texas, United States 


\title{
Trade-offs in the visible spectrograph of the ELT instrument MOSAIC
}

\author{
A.W. Janssen ${ }^{\mathrm{a}}$, E. Sokolova ${ }^{\mathrm{b}}$, J. Pragt ${ }^{\mathrm{a}}$, J. Kragt ${ }^{\mathrm{a}}$, Ramon Navarro ${ }^{\mathrm{a}}$, Niels Tromp ${ }^{\mathrm{a}}$, E.

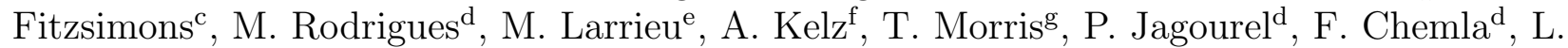 \\ Kaper $^{\mathrm{h}}$, and G. Dalton ${ }^{\mathrm{i}}$ \\ a NOVA Optical Infrared Instrumentation Group at ASTRON, P.O. Box 2, 7990 AA, \\ Dwingeloo, The Netherlands \\ ${ }^{\mathrm{b}}$ RiverD International B.V., Rotterdam, The Netherlands \\ ${ }^{\mathrm{c}}$ UK Astronomy Technology Centre, Science and Technology Facilities Council, Royal \\ Observatory Edinburgh, Blackford Hill, Edinburgh, EH9 3HJ, United Kingdom \\ ${ }^{d}$ GEPI, Observatoire de Paris, PSL research university, CNRS, Place Jules Janssen, 92195 \\ Meudon, France \\ eIRAP, Université de Toulouse III-CNRS, 9, avenue du Colonel Roche, BP 44346 - 31028 \\ Toulouse Cedex 4 \\ ${ }^{\mathrm{f}}$ AIP Leibniz-Institut f'ur Astrophysik Potsdam, An der Sternwarte 16, 14482 Potsdam, \\ Germany \\ ${ }^{\text {g} D u r h a m ~ U n i v e r s i t y-~ C f A I ~, ~ D e p a r t m e n t ~ o f ~ P h y s i c s, ~ S o u t h ~ R o a d, ~ D u r h a m ~ D H 1 ~ 3 L E, ~ U n i t e d ~}$ \\ Kingdom \\ ${ }^{\mathrm{h}}$ Anton Pannekoek Institute for Astronomy, Science Park 904, 1098 XH Amsterdam, The \\ Netherlands \\ ${ }^{\mathrm{i} U n i v e r s i t y ~ o f ~ O x f o r d, ~ A s t r o p h y s i c s ~ D e n y s ~ W i l k i n s o n ~ B u i l d i n g ~ K e b l e ~ R o a d ~ O x f o r d ~ O X 1 ~ 3 R H, ~}$ \\ United Kingdom
}

\begin{abstract}
MOSAIC is a concept for a multi-object spectrograph for the Extremely Large Telescope (ELT). It is planned to cover the wavelength range from $460 \mathrm{~nm}$ to $1800 \mathrm{~nm}$ with two types of spectrographs, optimized for the visible and the near-infrared. There are two observing modes; multiplex mode with 200 sampling points and Integral Field Unit (IFU) mode with 10 fields. The instrument consists of 5 visible spectrographs and 5 near-infrared spectrographs. The ELT is far from diffraction limited in the visible wavelength range. Rather than developing a large and complex AO system, it was decided that the instrument will be seeing limited in the visible. Spot sizes are therefore about $2.8 \mathrm{~mm}$ in diameter in the ELT focal plane, and need to be sampled by multiple fibers with large core diameter. As a result, large optics is required to achieve the science requirements on spectral resolution, bandwidth and multiplex. We work in close collaboration with manufacturers to design an instrument that is feasible and meets the scientific requirements.
\end{abstract}

Keywords: ELT, multi-object spectrograph, MOSAIC

\section{INTRODUCTION}

MOSAIC, the Multi-Object Spectrograph for Astrophysics, Intergalactic-medium studies and Cosmology, is an instrument concept for the multi-object spectrograph on the ESO Extremely Large Telescope (ELT-MOS). The current concept allows observation of 200 point sources or 10 IFUs in a wavelength range from $460 \mathrm{~nm}$ to $890 \mathrm{~nm}$ in the visible spectrographs, and simultaneously 100 point sources +100 background fibers or 10 Integral Field

Send correspondence to ajanssen@astron.nl

Ground-based and Airborne Instrumentation for Astronomy VII, edited by Christopher J. Evans, Luc Simard, Hideki Takami, Proc. of SPIE Vol. 10702, 107029F · @ 2018 SPIE · CCC code: 0277-786X/18/\$18 · doi: 10.1117/12.2313302 
Units (IFUs) in a wavelength range from $890 \mathrm{~nm}$ to $1800 \mathrm{~nm}$ in the near-infrared spectrographs. The phase A study successfully finished in March 2018, and phase B is expected to start in 2019

The science cases of a visible/near-infrared MOS at the ELT include spectroscopy of distant galaxies, evolution of large-scale structures, mass assembly galaxies through cosmic time, Active Galactic Nuclei/galaxy co-evolution, resolved stellar populations beyond the local group, galaxy archaeology, galactic center and plante formation. These topics are extensively discussed in the MOSAIC white paper and updates ${ }^{1}{ }^{2}$

MOSAIC is a combination of earlier instrument concepts EAGLE, ${ }^{4}$ Optimos EVE ${ }^{89}$ and Optimos Dioramas. ${ }^{5}$ Those studies have converged to the MOSAIC system design. Input parameters for the spectrograph design follow from design choices at the system level, including trade-offs that have been made on the focal plane design, field of view, background subtraction and sampling in the focal plane. ${ }^{3}$ It was decided to do the sampling with fibers, because of the various observing modes, the high multiplex and because the focal plane and the spectrographs are separated from each other by several meters. Especially the trade-off on number of fibers and fiber size are of importance for the visible spectrograph design ( described in section 2 ).

\section{ELT FOCAL PLANE SAMPLING}

Because of the large ELT focal length of about 680 meter, the plate scale in the ELT focal plane is only $0.3 \mathrm{l} / \mathrm{mm}$. This is a factor 5.5 smaller than the plate scale at the VLT, while the F-ratio of both telescopes is similar. Therefore, spot sizes at the ELT will be about 5.5 times larger in diameter than at the VLT, for similar seeing conditions and $\mathrm{AO}$ performance.

The performance of the ELT Ground Layer Adaptive Optics (GLAO) in the visible is uncertain at the moment, but is expected to be close to seeing limited. Most ELT instruments have an additional AO system tuned for the wavelength range of interest. The MOSAIC consortium also plans to enhance AO performance for the near-infrared observations, but not for the observations in the visible, since this would require an AO system that works down to $460 \mathrm{~nm}$ over a 7.4 arcminute FoV. This is far off from current state-of-the-art AO systems like SAXO ( for SPHERE at the VLT), GPI and MagAO which typically correct a 1-2 arcsecond FoV. The AO system for Micado, MAORY, plans to be diffraction limited at $800 \mathrm{~nm}$ at the ELT over $\sim 1$ arcminute FoV. In principle it is possible to increase the FoV and make it work at shorter wavelengths, but such a system would be very expensive. Moreover, on top of the AO correction, an atmospheric dispersion corrector would then be required to reduce the spot size. The atmospheric dispersion is largest in the visible, as shown in Figure 1. Considering the added complexity of adding an AO system that performs well in the visible and an Atmospheric Dispersion Corrector, it was decided to keep the instrument seeing-limited in the visible.

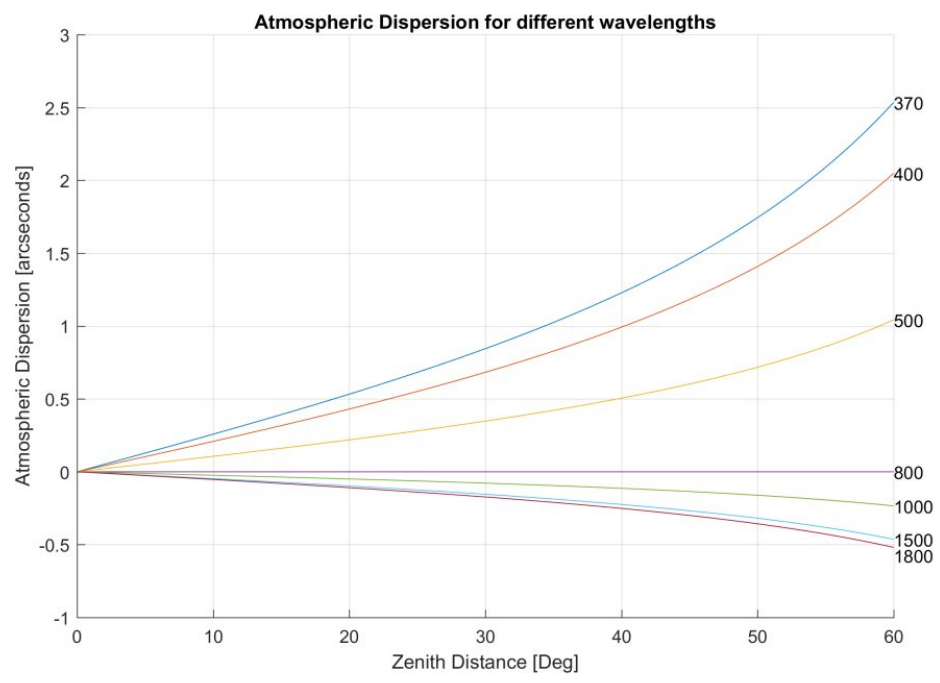

Figure 1. Atmospheric dispersion at Paranal 

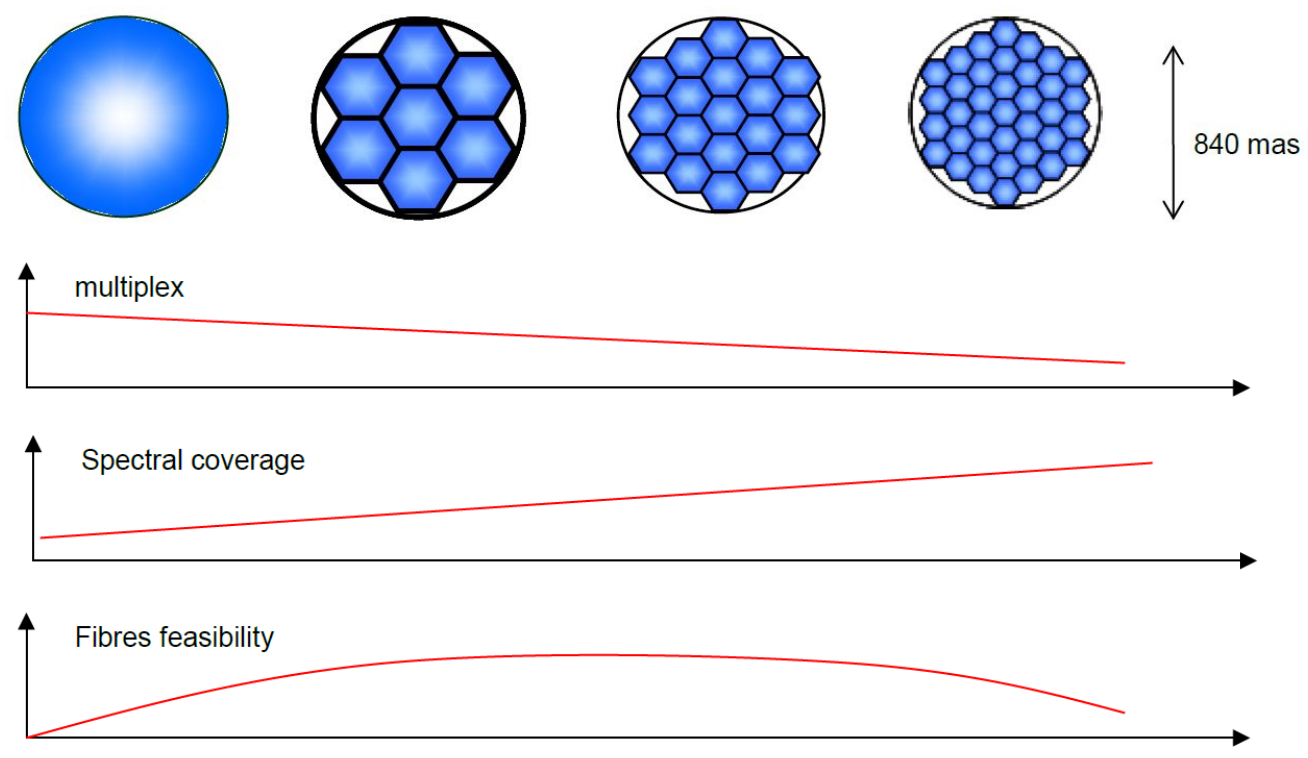

Spectrograph optics feasibility

Figure 2. Advantages and disadvantages of sampling with 1, 7, 19 and 37 fibers. A larger value on the y-axis corresponds to a larger multiplex, larger spectral coverage, better fiber feasibility, and better spectrograph optics feasibility.

Therefore typical median seeing conditions at Paranal - 0.64 arcsecond in the optical and 0.43 arcsecond in the near-IR - set the spot size in the ELT focal plane. For a circular aperture, the optimal diameter is around 0.9 arcsecond in the optical. There is some margin to reduce or increase these sizes, to allow some freedom in the optical design, without affecting the signal-to-noise ratio resulting in optimised on-sky apertures of 0.84 arcsecond. Note that these calculations will be revised to verify the design choice once better GLAO Point Spread Function simulations become available. With the ELT plate scale of 0.3 arcsecond $/ \mathrm{mm}$ this implies a $2.8 \mathrm{~mm}$ spot size. This spot is sampled with multiple microlenses that focus the light in fibers. The number of fibers and the fiber core size is a trade off. Several configurations have been studied, and the trades were performed on the following criteria: The multiplex per spectrograph, the slit width (directly conditioning the spectral resolution, so spectral coverage for a given detector size), the fibre manufacturing feasibility and the spectrograph optics size (also conditioned by the slit width and the required resolution). Assuming all options cover 840 mas and have microlenses with the same f-ratio, the approximate fiber core diameters are 845 micron, 280 micron, 170 micron and 120 micron for a total of 1, 7, 19 and 37 fibers respectively.

Using only one fibre is unfeasible in terms of fibre size and consequent slit width. The 7 fibres configuration is feasible in terms of microlenses and fibres, but leads to high constraints on the spectrograph optics. The next option (19 fibres) still allows a multiplex of 40 per spectrograph, without reaching the limits of the fibres feasibility. A configuration with 37 fibres reduces the multiplex per spectrograph too much. The configuration with 19 fibres is therefore adopted as a baseline.

\section{REQUIREMENTS}

The focal plane sampling sets the fiber core diameter and the f-ratio at the input and output (after Focal Ratio Degradation) of the fibers. The fiber core $(168.9 \mu \mathrm{m})$ and output F-ratio (3.64) is the same for High Multiplex Mode (HMM; 200 point sources) and Inter Galactic Medium Mode (IGMM; 10 IFUs of 2 arcsecond by 2 arcsecond in size ), in order to allow a single spectrograph design with similar performance for both modes. 
Secondly, it is preferred to have one detector per spectrograph, to avoid gaps in spectra and to minimize cost. Moreover a square detector makes better use of the image quality than a rectangular detector, because image quality is usually best in the center and drops with radial distance to the center. The detector is therefore a single CCD of $90 \mathrm{~mm}$ by $90 \mathrm{~mm}$ in size with 15 micron pixels. With a fiber diameter $>10$ times the pixel size, a fast camera is required to reduce the projected fiber diameter. An F-ratio of about 1.6 is a good compromise between projected fiber size and realistic optical components. This results in a resolution element of 4.2 pixels ( 0.85 times the projected fiber core diameter).

The pupil diameter $D$, incidence angle $\gamma$ (in Littrow mode) and line spacing of the gratings $d_{s}$ is calculated as follows, where $d_{f}$ is the actual fiber size and $F \#$ the collimator F-ratio. The effective resolution element of a circular fiber is 0.85 times the fiber diameter.

$$
\begin{gathered}
R=2 \tan \gamma \frac{F \# \cdot D}{0.85 \cdot d_{f}} \\
d_{s}=\frac{\lambda}{2 \sin \gamma}
\end{gathered}
$$

A large spectral resolution can be achieved by increasing the pupil diameter or the grating line density, but both have practical limits. A pupil diameter of $19 \mathrm{~cm}$ ensures the gratings are manufacturable both in terms of size and line density.

The input parameters and requirements to the spectrograph design are:

- $\mathrm{R}=5000$ from $460 \mathrm{~nm}$ to $890 \mathrm{~nm}$, covered by 3 gratings/gratings

- $\mathrm{R}=15000$ for a small wavelength range around $650 \mathrm{~nm}$ and $860 \mathrm{~nm}$

- fibre core is $168.9 \mu \mathrm{m}$ in both modes

- fibre output ratio and collimator F ratio are 3.64 in both modes

- camera $\mathrm{f}$ ratio is 1.6

- detector size is $90 \mathrm{~mm}$ by $90 \mathrm{~mm}$

- slit length is $204.5 \mathrm{~mm}$ (follows from detector size and $\mathrm{F}$ ratios)

The slit length follows from the detector size, camera F ratio and collimator F ratio. However, with a $20 \mathrm{~cm}$ long slit the multiplex of one such spectrograph is 40, while the goal is to accomodate 200 objects. Therefore 5 identical visible spectrographs are foreseen. The number of spectrographs can be reduced if downscaling is necessary. Table 1 lists the number of objects and fibers per spectrograph.

\begin{tabular}{lll}
\hline & HMM & IGMM \\
\hline Total \# of objects & 200 & 10 \\
\# objects/spectrograph & 40 & 2 \\
\# fibers/object & 19 & 221 \\
\# fibers/slit & 760 & 442 \\
\hline
\end{tabular}

Table 1. Number of objects and fibres per spectrograph, both in HM Mode and IGM Mode, for a total of 5 spectrographs. 


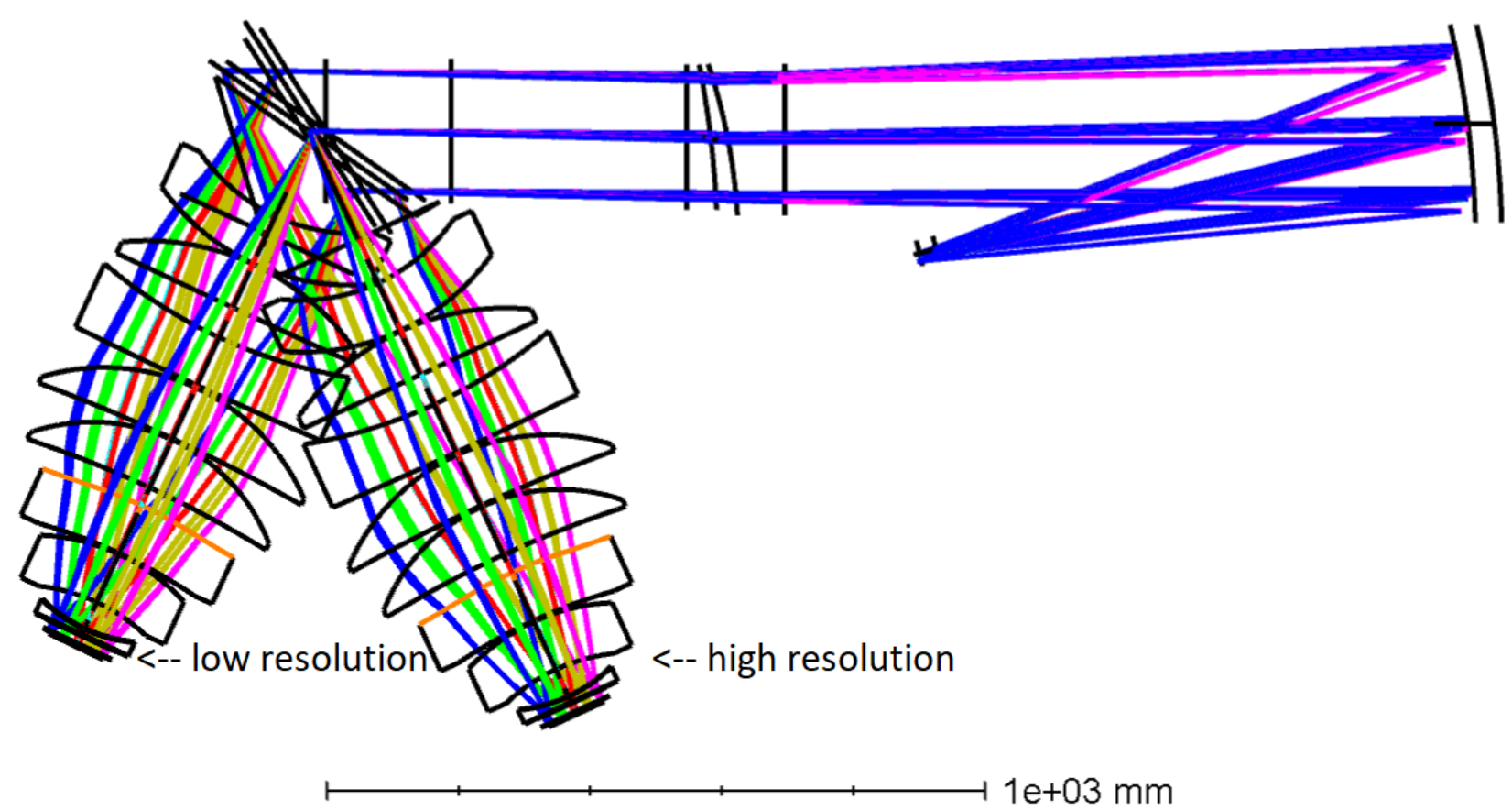

Figure 3. Optical layout with light entering through the slit, to the collimator, 2 corrector lenses, grating, 8 camera lenses, and detector. Both the high spectral resolution mode and the low spectral resolution mode are shown.

\section{OPTICAL DESIGN}

The optical design for the MOSAIC visible spectrographs is based on the experience with Optimos Eve E78 $^{67}$ (together with EAGLE and Optimos Dioramas a precursor to MOSAIC) and WEAVE. ${ }^{10}$ The design is optimized for throughput and feasibility. This results in the selection of VPH gratings because of their larger efficiency than reflection gratings, and spherical surfaces where possible to facilitate the manufacturing and alignment.

The chosen design is a classical spectrograph with an off-axis reflective collimator, VPH grating and transmissive camera. A transmissive camera allows on-axis focusing of the dispersed beam without obscuration by the detector. Figure 3 shows the layout with 2 possible positions for the camera, which correspond to the high spectral resolution mode and the low spectral resolution mode. All lenses and the mirror are on-axis spheres, except for the corrector lenses which are off-axis spheres and camera lens 6 which is a weak asphere. The largest camera lens has a $440 \mathrm{~mm}$ mechanical diameter. We have contacted Schott to discuss the availability of the lenses. The maximum lens diameter depends on the material, but lenses with diameters $>250 \mathrm{~mm}$ are typically hard to produce. Since most lenses in the camera have diameters $>250 \mathrm{~mm}$, the choice of material is limited. The current design includes fused silica, N-BK7, F2, SF6, SF57, SF5, and N-SF6 lenses, based on information from Schott about what is available. Alternatives are currently being explored with other manufacturers.

The fiber core diameter dominates the spot size at the detector. It has a projected diameter of 74 micron, compared to 10-20 micron RMS radius caused by the optical aberrations of the system. Because of this spot size a large dispersion is needed in order to reach a spectral resolution of 5000 and 15000 . This is achieved by VPH gratings with the line density and dimensions as listed in Table 2. Rather high incidence angles are required especially for the $\mathrm{R}=15000$ bands. This is achieved by letting the light enter and exit the VPH through fused silica prisms, see Figure 5. This way it is possible to have a 55 degree incidence angle in fused silica, which would correspond to $>90$ degrees in air. A disadvantage of this configuration with large incidence angle and line density (apart from the additional mass of the prisms) is the narrow throughput curve (Figure 4). Therefore the high resolution gratings are still under consideration. 


\begin{tabular}{lllll}
\hline Description & Length $[\mathrm{mm}]$ & Lines/mm & Angle (deg) & Prism material \\
\hline LR 525 & 223 & 2057 & 32 & none \\
LR 634 & 223 & 1665 & 32 & none \\
LR 800 & 223 & 1352 & 32 & none \\
HR 656 & 336 & 3730 & 55 & Fused silica \\
HR 862 & 336 & 2840 & 55 & Fused silica \\
\hline
\end{tabular}

Table 2. VPH parameters: central wavelength, length, line density, incidence angle and prism material.

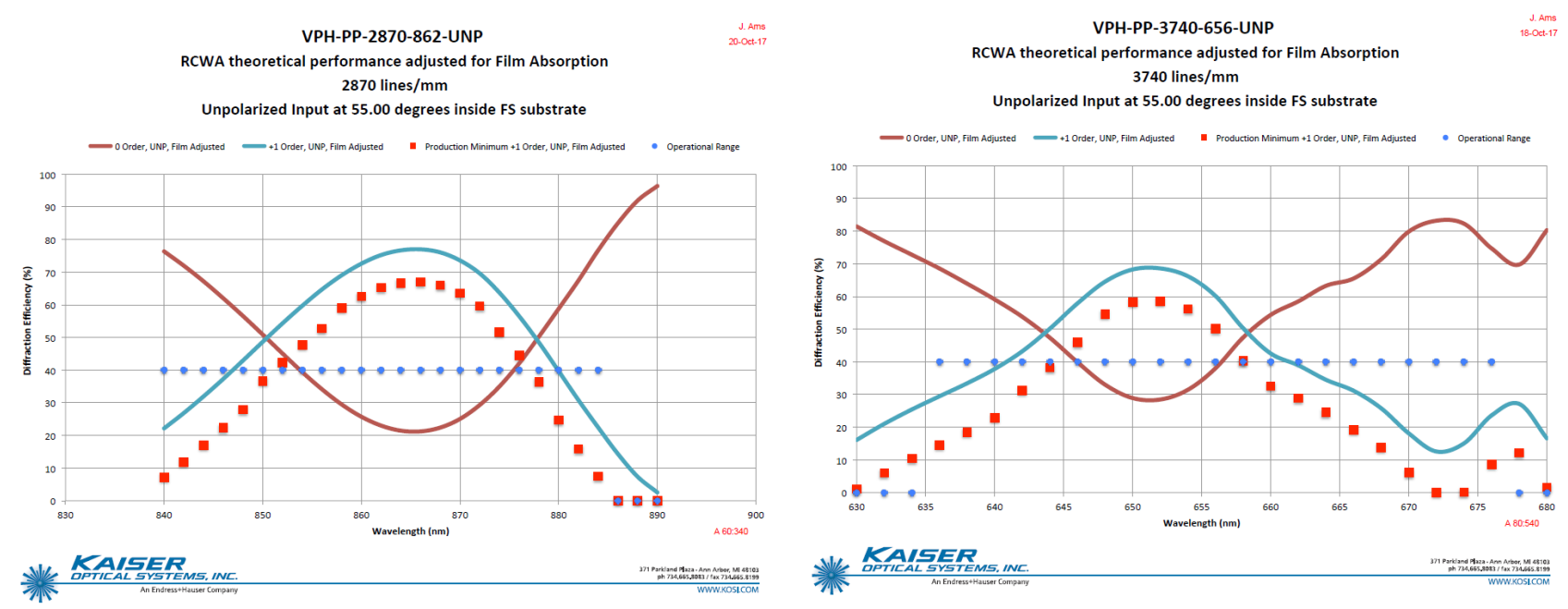

Figure 4. Theoretical and expected throughput in first order of the R 15000 resolution gratings, centered at $656 \mathrm{~nm}$ and $862 \mathrm{~nm}$. The narrow throughput curve is a result of the grating's large line density.

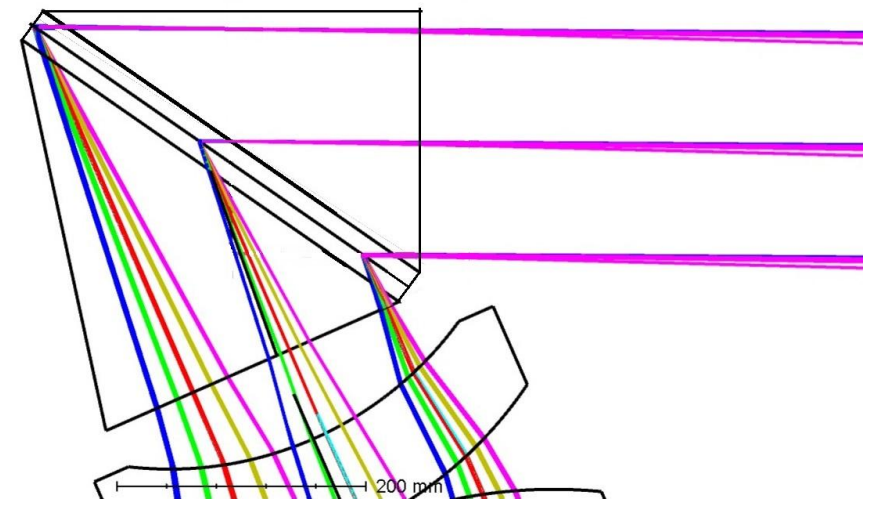

Figure $5 . \mathrm{VPH}+2$ prisms + first camera lens as used in the HR bands. The prisms are required to enable an incidence angle of 55 degrees within fused silica. 


\section{ALTERNATIVES}

Throughput was the main design goal, followed by feasibility of the manufacturing of optical components and reducing the size of the instrument. Despite measures to keep the individual parts small, the overall instrument (the focal plane unit, the 5 visible spectrographs, 5 near-infrared spectrographs and electronics racks) exceeds the mass- and volume- allocations. Ways to overcome that issue are being investigated with ESO. A significant reduction in the visible spectrograph size requires shifting of design priorities or changing requirements. Some potential alteratives to the current design are described in this section.

The number of camera lenses may be reduced by adding aspherical surfaces. Although aspheres add complexity, this method may make the overall design simpler if large lenses can be removed. It will however not change the overall scale of the optics.

A reflective on-axis camera, like in MOONS, ${ }^{11}$ has been considered. This might even allow for a faster camera, reducing the spot size at the detector, and hence relaxing the dispersion requirements on the gratings. The main disadvantage is the large obscuration in the center of the beam. Even with an F/1 camera the detector would have to be $60 \mathrm{~mm}$ large, hence forming a large obscuration.

It has recently become possible to produce curved detectors, potentially even at sizes as large as $90 \mathrm{~mm}$ by 90 $\mathrm{mm}$. A curved detector greatly simplifies the optical design of the camera. In the ideal case, the camera could be removed entirely and the focusing is done by a curved reflective grating projecting on a curved CCD. In practice it will be difficult to produce a curved grating with the required size and spectral resolution. More realistically, the number of lenses in the camera could be reduced and potentially a faster camera can be achieved. Preliminary models suggest that 4 or 5 lenses can be removed from the camera, which would improve the throughput with 5 to $10 \%$ (assuming 0.5 to $1 \%$ loss per surface) and reduce the camera size. The main disadvantage is that curved CCDs are relatively new and therefore risky and costly.

Reflective gratings have lower efficiency than VPH gratings, but may allow for a more efficient use of space especially when the collimator is used in double-pass. This requires the collimator and camera have the same F-ratio, hence a significant modification of the design.

\section{REFERENCES}

[1] Evans et al, "Science case and requirements for the MOSAIC concept for a multi-object spectrograph for the European Extremely Large Telescope", proc SPIE 9147, 9147E..96E, 2014

[2] Evans et al., "Science requirements and trade-offs for the MOSAIC instrument for the European ELT", proc SPIE 9908, 9908E..9JE, 2016

[3] Rodrigues et al. "Developing an integrated concept for the E-ELT Multi-Object Spectrograph (MOSAIC): design issues and trade-offs", proc SPIE, 9908, 9908E..9SR, 2016

[4] Cuby et al, "EAGLE: a MOAO fed multi-IFU NIR workhorse for E-ELT", proc SPIE 7735, 7735E..2DC, 2010

[5] Le Fevre et al, "OPTIMOS-DIORAMAS: A Wide-field Imaging and Multi-slit Spectrograph for the E-ELT", The Messenger, 140 34L, 2010

[6] Spanò et al., "OPTIMOS-EVE optical design of a very efficient, high-multiplex, large spectral coverage, fiber-fed spectrograph at EELT", proc SPIE 7735, 7735E..6PS, 2010

[7] Chemla et al., "OPTIMOS-EVE design trade-off analysis", proc SPIE 7735, 7735E..5LC, 2010

[8] Navarro et al., "Project overview of OPTIMOS-EVE: the fibre-fed multi-object spectrograph for the E-ELT", proc SPIE 7735, 7735E..2LN, 2010

[9] Hammer et al, "OPTIMOS-EVE: A Fibre-fed Optical-Near-infrared Multi-object Spectrograph for the EELT", The Messenger, 140 36H, 2010

[10] Rogers et al., "The design of the WEAVE spectrograph", proc SPIE 9147, 9147E..6HR, 2014

[11] Oliva et al. "Updated optical design and trade-off study for MOONS, the Multi-Object Optical and Near Infrared spectrometer for the VLT", proc SPIE 9147, 9147E..2CO, 2014 